\title{
Molecular Characterization of Fluoroquinolone Resistance of Methicillin- Resistant Clinical Staphylococcus aureus Isolates from Rawalpindi, Pakistan
}

\author{
Bernard S Marasa ${ }^{a}$, Saira Iram ${ }^{\mathrm{b}}$, Kidon Sung ${ }^{\mathrm{a}}$, Ohgew Kweon ${ }^{\mathrm{a}}$, Carl E Cerniglia ${ }^{\mathrm{a}}$ \& Saeed Khan ${ }^{\mathrm{a}^{*}}$
}

\begin{abstract}
Division of Microbiology,

National Center for

Toxicological Research, US

Food and Drug Administration, Jefferson, AR 72079, USA ${ }^{\text {a. }}$,

Microbiology Research

Laboratory, Department of

Microbiology, Quaid-I-Azam

University, Islamabad 45320,

Pakistan ${ }^{\mathrm{b}}$.

Keywords: Quinolone

Resistance Determining

Region, Methicillin-Resistant

Staphylococcus aureus, Efflux

Pump Inhibitors, gyrA, grlA,

norA

* Corresponding Author:

Saeed A Khan, Ph.D.

Tel: +1 8705437197

Fax: +1 8705437307

E-mail:

saeed.khan@fda.hhs.gov

Date of submission of

manuscript: 18-06-2015

We have characterized 10 multidrug-resistant Staphylococcus aureus clinical isolates from Pakistan, with respect to their fluoroquinolone resistance, mutations in $\operatorname{gr} \mathrm{r} / \mathrm{B}$, and $\mathrm{grlA} / \mathrm{B}$ genes, and the presence and expression of the efflux pump genes nor A, norB, norC, and mdeA. All the isolates were highly resistant to ciprofloxacin, enrofloxacin, levofloxacin, and norfloxacin and possessed one or more efflux pump genes. The efflux pumps, along with the single and double point mutations S84L, S84L and G106D observed in gyrA; and S80Y, S81P, $\mathrm{E} 84 \mathrm{G}$, and $\mathrm{S} 80 \mathrm{~F}$ and $\mathrm{E} 84 \mathrm{G}$ in grlA genes; enhanced fluoroquinolone resistance in these isolates. A single deletion of nucleotide "A" upstream of the putative Fur-binding box and the presence of two novel mutations, G291D in isolate 30, 32, 35, and 41 and V371I in isolate 10 within the norA coding region, were also observed. An in silico structural analysis revealed an increase in Nor $A_{G 291 D}$ stability with a predicted $\Delta \Delta \mathrm{G}$ of 0.48 $\mathrm{kcal} / \mathrm{mol}$, but a decrease in Nor $A_{\mathrm{V} 371 \mathrm{I}}$ stability by $-1.12 \mathrm{kcal} / \mathrm{mol}$. Moreover, an increased transcriptional expression of the norA, norB, norC, and mdeA genes and relatively higher fluoroquinolone resistance in isolates with a G291D mutation, compared to that of the isolate with a V371I mutation, suggests a possible role of these mutations in fluoroquinolone resistance. The data provide new insights into the mechanism of fluoroquinolone resistance and may lead to an enhanced understanding of multidrug resistance observed in MRSA isolates and the development of effective mitigation strategies.
\end{abstract}

Accepted on: 04-07-2015

\subsection{Introduction}

Staphylococcus aureus remains one of the most important bacterial pathogens in the public health domain due to its high virulence and capability to cause multiple ailments ranging from complicated skin and skin structure infections (cSSSI), to life threatening conditions, such as endocarditis, pneumonia, and toxic shock syndrome $[11,21]$. This pathogen is relatively abundant both in the community and healthcare facilities [7]. Besides its pathogenic potential, this gram-positive bacterium has been able to develop resistance to almost all classes of antibiotics, starting in the 1950s, followed by resistance to methicillin a decade later due to the activity of PBP2a protein, encoded by the mecA gene of staphylococcal cassette chromosome mec (SCCmec) mobile genetic element [2]. For some time now the fluoroquinolone (FQ) 
class of antibiotics and vancomycin has remained a common and effective therapy against $S$. aureus. However, mutations in the quinolone resistance determining region (QRDR) of genes encoding the target enzymes, gyrase and/or topoisomerase IV have led to higher resistance to FQs [22].

Other than mutations in the QRDR hotspots, the presence of efficient efflux pumps, which extrude drugs or other noxious agents from these bacterial cells, enhances resistance to FQ $[6,8,25]$. Mutations within the promoter region of the multidrug efflux pump gene norA have been shown to directly affect the over-expression of the norA efflux pump in $S$. aureus. Very little information is available about the level of FQ resistance and the underlying resistance mechanism(s) in clinical S. aureus isolates from Pakistan. The present study analyzes the genetics of FQ resistance by analyzing the mutations in QRDRs of the target genes gyrA, gyrB, grlA, grlB, norA and the promoter region of norA in FQ-resistant human clinical MRSA isolates from Rawalpindi, Pakistan. In this study, we have shown that the FQ-resistant MRSA isolates, apart from carrying wellknown mutations that confer FQ resistance, also carry some novel mutations, deletion, and an enhanced activity of several efflux pump genes, such as norA, norB, nor $C$, and $m d e A$, which help them survive and develop high resistance to FQs.

\subsection{Materials and methods}

\subsection{Bacterial strains}

Ten nasal and perirectal swabs of patients were procured from a tertiary health care facility in Rawalpindi, Pakistan after obtaining an import permit from the Center for Disease Control, USA. These were initially characterized as $S$. aureus, based on their $n u c A$ and coagulase profiles. They were further subjected to VITEK testing at the FDA's National Center for Toxicological Research (NCTR) and confirmed as $S$. aureus isolates. Methicillin-resistance of these organisms was determined by oxacillin
(10 $\mu \mathrm{g} / \mathrm{ml})$ disk diffusion assays on $\mathrm{MH}$-agar plates and confirmed by PCR detection of the mecA gene. A quality control strain of $S$. aureus subsp. aureus (ATCC ${ }^{\circledR}$ 25923) was purchased from the American Type Culture Collection (Manassas, VA, USA).

\subsection{Antimicrobial susceptibility testing and MIC determination}

Erythromycin, tetracycline, ampicillin, van comycin, and chloramphenicol were purchased from Sigma-Aldrich (St. Louis, MO, USA) and the FQs (enrofloxacin, nor floxacin, ciprofloxacin and levofloxacin) were purchased from ICN Biomedicals, Inc. (Aurora, OH, USA). Antibiotic-coated discs containing ampicillin $(10 \mu \mathrm{g} / \mathrm{ml})$, cefixime (5 $\mu \mathrm{g} / \mathrm{ml})$, ciprofloxacin $(5 \mu \mathrm{g} / \mathrm{ml})$, erythromycin $(15 \mu \mathrm{g} / \mathrm{ml})$, gentamicin $(120 \mu \mathrm{g} / \mathrm{ml})$, kanamycin $(30 \mu \mathrm{g} / \mathrm{ml})$, levofloxacin $(5 \mu \mathrm{g} / \mathrm{ml})$, norfloxacin $(10 \mu \mathrm{g} / \mathrm{ml})$, oxacillin $(10 \mu \mathrm{g} / \mathrm{ml})$, penicillin $(10 \mathrm{U})$, and tetracycline $(30 \mu \mathrm{g} /$ $\mathrm{ml}$ ), were acquired from Remel Fisher Scientific (Lenexa, KS, USA). Susceptibility of $S$. aureus isolates to antibiotics was determined using the Kirby-Bauer disc diffusion method [1] on MH-agar plates at $37^{\circ} \mathrm{C}$. MICs were determined by the broth microdilution method following CLSI guidelines [3].

\subsection{Effect of efflux pump inhibitors (EPI) on MIC}

The EPIs, including carbonyl cyanide- $m$ chlorophenylhydrazone (CCCP) and reserpine (RES) were purchased from Sigma-Aldrich. RES was dissolved in DMSO, while CCCP was prepared in 50\% methanol: water $(\mathrm{v} / \mathrm{v})$. All solutions were prepared fresh and protected from light. The EPIs used in this study were evaluated for their ability to reduce resistance to FQ antibiotics, using a previously published method [23]. The assay was based on the principle that if bacteria are highly resistant to an antibiotic due to the presence of an efflux pump, inhibition of the efflux pump activity will stop the antibiotic from being 
forced out of the cell and hence a lesser amount of antibiotic will be required to kill the bacteria. This will result in lowering the MIC value of the antibiotic [20]. Using this principle, we measured the MIC of FQ antibiotics in the absence and presence of CCCP and RES at $0.36 \mathrm{mg} / \mathrm{L}$ and $5 \mathrm{mg} / \mathrm{L}$, respectively. The concentrations of the CCCP and RES used here were $50 \%$ of their MIC and did not inhibit the growth of bacteria in media. Bacterial growth was measured by recording the $\mathrm{OD}_{600}$ using a microplate reader (BioTek Instruments, Inc., Winooski, VT, USA) programmed to record the readings every $15 \mathrm{~min}$ for $18 \mathrm{~h}$. All assays were performed in triplicate unless indicated otherwise.

\subsection{Effect of efflux pump inhibitors (EPI) on ethidium bromide $(E t B r)$ fluorescence}

Efflux pump activity was monitored by the $\mathrm{EtBr}$ uptake and measurement of the fluorescence in the presence and absence of EPIs as described previously [15] with some modifications. The bacteria were grown overnight at $37^{\circ} \mathrm{C}$ in $\mathrm{LB}$ broth containing $1 \%$ sodium chloride and the cells were centrifuged for $5 \mathrm{~min}$ at $18,000 \mathrm{X} \mathrm{g}$ in an Eppendorf centrifuge (Centrifuge 5417C, rotor F45-30-11) (Eppendorf North America, Hauppauge, NY, USA). The cell pellets were washed two times with $50 \mathrm{mM}$ sodium phosphate buffer ( $\mathrm{pH}$ 7.0) containing $0.2 \%$ glucose and resuspended in the same buffer. The optical density at $600 \mathrm{~nm}$ was adjusted to 0.4 and $100 \mu \mathrm{l}$ amounts of cell suspensions were transferred into the wells of black 96-well microtiter plates (Corning, Inc., Life Sciences, Tewksbury, MA). EtBr, at a final concentration of $2.5 \mathrm{mg} / \mathrm{L}$, was added and the fluorescence readings were recorded in a Synergy 2 Multi-Mode Microplate Reader. Fluorescence was read every min with excitation at $530 \mathrm{~nm}$ and emission at $600 \mathrm{~nm}$. EtBr fluorescence was also recorded in the presence of CCCP and RES at final concentrations of $0.36 \mathrm{~g} / \mathrm{L}$ and 5 $\mathrm{g} / \mathrm{L}$, respectively. The natural fluorescence of the cells was subtracted, and the fluorescence intensity was expressed in relative fluorescence units (RFU). All experiments were performed in triplicate. If the efflux pump were inhibited by CCCP and/or RES, the fluorescence intensity would be higher compared to when it was uninhibited.

\subsection{Isolation of chromosomal DNA}

Chromosomal DNA was isolated from $S$. aureus isolates grown overnight in $\mathrm{MH}$ broth at $35^{\circ} \mathrm{C}$. Bacterial cells $(1 \mathrm{ml})$ were centrifuged at 10,000 X $g$ and the pellet was suspended in $180 \mu \mathrm{l}$ of TE buffer $(10 \mathrm{mM}$ Tris- $\mathrm{HCl}+1 \mathrm{mM}$ EDTA, $\mathrm{pH}$ 8.0) containing 10 units of lysostaphin (Sigma-Aldrich). The microfuge tubes containing this suspension were incubated at $37^{\circ} \mathrm{C}$ for $1 \mathrm{~h}$ followed by the addition of proteinase K (Sigma-Aldrich) and incubation at $55^{\circ} \mathrm{C}$ for $1 \mathrm{~h}$. After this step, $200 \mu \mathrm{l}$ of AL solution, made by mixing AL1 and AL2 reagents from QIAmp kit (Qiagen, Valencia, CA) was added to the tube. Further processing for the purification of DNA from $S$. aureus isolates was done as per manufacturer's instructions. The DNA concentration was determined by measuring the absorbance at 260 and $280 \mathrm{~nm}$ in a Nanodrop 2000 spectrophotometer (Thermo Fisher Scientific, Wilmington, DE, USA). Plasmid DNA preparations were done with a Qiagen Plasmid Midi or Maxi kit, according to the manufacturer's protocol, but with specific modifications for Staphylococcus. For $S$. aureus, the procedure was modified by the inclusion of lysostaphin (SigmaAldrich) at a final concentration of 100 $\mu \mathrm{g} / \mathrm{ml}$ in cell lysis buffer and the elution buffer was warmed to $65^{\circ} \mathrm{C}$ for the elution of plasmid DNA from the Qiagen cartridge. DNA was analyzed on $1 \%$ agarose gels by electrophoresis at $1 \mathrm{~V} / \mathrm{cm}$ in $1 \mathrm{X}$ TBE buffer followed by staining with $\mathrm{EtBr}(1 \mu \mathrm{g} / \mathrm{ml})$ in electrophoresis buffer and photography by gel documentation system GDS7500 (UVP, Inc., Upland, CA, USA).

\subsection{PCR amplification of $F Q$ resistance determinant and efflux pump genes}

PCR amplification of the $\operatorname{grlA}, \operatorname{grlB}, \mathrm{gyrA}$, and $\operatorname{gyr} B$, the norA gene promoter region, 
and the norA genes was carried out in a GeneAmp PCR system (Bio-Rad, Hercules, CA, USA) using previously published cycling conditions $[6,18,31]$ as summarized in supplementary table S1. The PCR reactions were performed in a total volume of $50 \mu \mathrm{l}$, which contained $1.6 \mathrm{mM}$ each deoxynucleoside triphosphate (Applied Biosystems, Foster City, CA, USA), 20 pmol of each primer (Table S1), $1 \mathrm{mM} \mathrm{MgCl}_{2}, 0.5$ $\mathrm{U}$ of AmpliTaq Gold Enzyme (Applied Biosystems), and $0.5 \mu \mathrm{l}$ of genomic DNA ( $500 \mathrm{ng}$ of DNA) in $1 \mathrm{X}$ buffer. After the PCR runs were over, the products were separated by electrophoresis on a $0.8 \%$ agarose gel. The gel was stained with $\mathrm{EtBr}(1.0 \mu \mathrm{g} / \mathrm{ml})$ and visualized using the GDS 7500 gel documentation system. Molecular weight markers (100 bp DNA ladder) were used to determine the size of the amplicons.

\subsection{RNA isolation}

The $S$. aureus isolates were grown in Luria Bertani (LB) broth (Sigma-Aldrich) at $37^{\circ} \mathrm{C}$ overnight and the next day, the optical density $\left(\mathrm{OD}_{600}\right)$ was adjusted to 0.5 by addition of fresh LB or LB supplemented with $2.5 \mathrm{mg} / \mathrm{L} \mathrm{EtBr}$. The bacteria were incubated further at $37^{\circ} \mathrm{C}$ for $1 \mathrm{~h}$. The bacterial cells were pelleted by centrifugation and washed once with TrisEDTA buffer. The cells were pelleted again, suspended in Tris-EDTA buffer containing $100 \mathrm{mg} / \mathrm{L}$ lysostaphin, followed by incubation for $15 \mathrm{~min}$ at $37^{\circ} \mathrm{C}$. RNA was isolated using Qiagen's RNeasy kit according to the manufacturer's protocol. Residual DNA was removed by treatment with 5 units of DNase I (Life Technologies, Grand Island, NY, USA) at $37^{\circ} \mathrm{C}$ for $45 \mathrm{~min}$ followed by inactivation at $50^{\circ} \mathrm{C}$ for $10 \mathrm{~min}$. The RNA concentration was determined by measuring the absorbance at 260 and $280 \mathrm{~nm}$ in a Nanodrop 2000 spectrophotometer (Wilmington, DE, USA) and 50 ng of RNA was reverse transcribed with Thermo Scientific First Strand cDNA Synthesis kit (Thermo Fisher Scientific Inc., Waltham, MA, USA). The cDNA was diluted five-fold for PCR amplification.

\subsection{RT-qPCR amplification of efflux pump genes}

Real-time PCR was performed in triplicate using the CFX96 Real-time PCR System (Bio-Rad) and the primers previously published [6, 18, 31] and listed in supplementary table 1 . The PCR reactions were carried out in a $20 \mu \mathrm{l}$ volume using Applied Bioscience's SYBR Green 2X Master mix kit according to the manufacturer's instructions. The reaction mix included $10 \mu \mathrm{l}$ of 2 X SYBR master mix, $8 \mu \mathrm{l}$ of five-fold diluted cDNA template from each isolate and DNase free water. The amplification cycling was carried out as follows; initial DNA denaturation at $95^{\circ} \mathrm{C}$ for $5 \mathrm{~min}$, followed by 39 cycles of denaturation at $95^{\circ} \mathrm{C}$ for $10 \mathrm{~s}$, $60^{\circ} \mathrm{C}$ annealing for $30 \mathrm{~s}$, and $72^{\circ} \mathrm{C}$ extension for $5 \mathrm{~s}$. The quantification data were analyzed with the Bio-Rad CFX Manager software, version 1.10. The relative quantity of mRNAs of the efflux pump genes was determined using the comparative threshold cycle $\left(C_{T}\right)$ method [19] with expression levels of $16 \mathrm{~S}$ rRNA used for normalization.Data were collected from three independent experiments.

\subsection{Sequence analysis and in silico structural analysis}

DNA amplicons specific for the $\operatorname{grlA}, \operatorname{grlB}$, gyrA, gyrB, norA promoter, and norA genes were purified by a QIAquick gel extraction kit and eluted in nuclease-free water. Both strands of the amplicons were sequenced by Sanger's dideoxy sequencing method [29]. Primers used for the amplification of these genes were used for the sequencing. For the detection of mutations, the sequences were aligned with known gene sequences in the GenBank database and compared using MegAlign software module of the DNAstar program (DNAstar Inc. Madison WI, USA). For computational modeling and analysis of the NorA efflux pump from $S$. aureus, the crystal structure of the YajR transporter [14] (PDB ID: 3WDO) from E. coli was used as a template. The homology 3D models for the wild-type and mutant proteins were 
generated using Phyre-2 [16]. The databases, RCSB PDB (http://www.rcsb.org/pdb/home /home.do) and PDB sum (http://www.ebi. ac.uk/pdbsum/) provided the experimentallydetermined PDB structure files and their structural overview. CUPSAT (http://cupsat. tu-bs.de) was adopted to predict changes in protein stability due to the point mutation. SCit (http://bioserv.rpbs.jussieu.fr/cgi-bin/S Cit) and CryCo5 (http://ligin.weizmann.ac. il/ lpge rzon/cryco5.0/cryco/cryst1.cgi) were used to identify clusters of the side chains of the mutants in contact in the structure. Determination of trans-membrane helices and extra-membrane loops was predicted by SOSUI [13] and TMHMM [17]. PyMOL (0.99RC6) (http://www.pymol.org) was used for visualization of structures.

\subsection{Results}

\subsection{Antimicrobial susceptibility and MIC}

Kirby-Bauer assays indicated that all MRSA isolates were resistant to multiple antibiotics, including ampicillin, penicillin, cefixime, ciprofloxacin, levofloxacin, enrofloxacin, norfloxacin, erythromycin, oxacillin, tetracycline, gentamicin, and kanamycin (Table 1). The MIC assays revealed that the isolates exhibited high levels of resistance to FQ with levofloxacin exhibiting the lowest MIC values (8-128 $\mathrm{mg} / \mathrm{L}$ ) among the four FQ antibiotics tested. Among all the isolates tested, isolate $10 \mathrm{~S}$ exhibited the lowest MICs for ciprofloxacin $(64 \mathrm{mg} / \mathrm{L})$, norflo-xacin (32 $\mathrm{mg} / \mathrm{L})$, and enrofloxacin (64 $\mathrm{mg} / \mathrm{L})$ compared to the other isolates. The MICs for other isolates of ciprofloxacin, norfloxacin, and enrofloxacin ranged between (128-512 $\mathrm{mg} / \mathrm{L}),(64-512 \mathrm{mg} / \mathrm{L})$, and (128-512 mg/L), respectively.

Table 1. Antimicrobial susceptibility profiles of clinical $S$. aureus isolates

\begin{tabular}{|c|c|c|c|c|c|c|c|c|c|c|c|c|c|}
\hline Isolate \# & Source & Coagulase & Catalase & $n u c A^{\mathrm{a}}$ & $\operatorname{Cip}^{a}$ & Nor ${ }^{a}$ & $\mathbf{L e v}^{\mathrm{a}}$ & Enr $^{\mathbf{a}}$ & Oxa ${ }^{a}$ & Tet $^{a}$ & Ery $^{a}$ & Gen $^{a}$ & $\operatorname{Kan}^{a}$ \\
\hline 10 & Nasal & + & + & + & $\mathrm{R}(64)$ & $\mathrm{R}(32)$ & $\mathrm{R}(32)$ & $\mathrm{R}(64)$ & $\mathrm{R}(512)$ & $\mathrm{R}$ & $\mathrm{R}$ & $\mathrm{R}$ & $\mathrm{R}$ \\
\hline $30 *$ & Nasal & + & + & + & $\mathrm{R}(128)$ & $\mathrm{R}(64)$ & $\mathrm{R}(32)$ & $\mathrm{R}(128)$ & $\mathrm{R}(512)$ & $\mathrm{R}$ & $\mathrm{R}$ & $\mathrm{R}$ & $\mathrm{R}$ \\
\hline 32 & Peri-rectal & + & + & + & $\mathrm{R}(512)$ & $\mathrm{R}(128)$ & $\mathrm{R}(8)$ & $R(256)$ & $\mathrm{R}(512)$ & $\mathrm{R}$ & $\mathrm{R}$ & $\mathrm{R}$ & $\mathrm{R}$ \\
\hline $35^{*}$ & Nasal & + & + & + & $\mathrm{R}(512)$ & $R(256)$ & $\mathrm{R}(128)$ & $R(256)$ & $R(512)$ & $\mathrm{R}$ & $\mathrm{R}$ & $\mathrm{R}$ & $\mathrm{R}$ \\
\hline $41^{*}$ & Peri-rectal & + & + & + & $R(256)$ & $R(256)$ & $\mathrm{R}(16)$ & $\mathrm{R}(512)$ & $\mathrm{R}(512)$ & $\mathrm{R}$ & $\mathrm{R}$ & $\mathrm{R}$ & $\mathrm{R}$ \\
\hline $42 *$ & Nasal & + & + & + & $R(256)$ & $\mathrm{R}(512)$ & $\mathrm{R}(64)$ & $\mathrm{R}(512)$ & $\mathrm{R}(512)$ & $\mathrm{R}$ & $\mathrm{R}$ & $\mathrm{R}$ & $\mathrm{R}$ \\
\hline $49^{*}$ & Nasal & + & + & + & $\mathrm{R}(512)$ & $\mathrm{R}(128)$ & $R(16)$ & $\mathrm{R}(128)$ & $R(512)$ & $\mathrm{R}$ & $\mathrm{R}$ & $\mathrm{R}$ & $\mathrm{R}$ \\
\hline 50 & NA & + & + & + & $R(256)$ & $\mathrm{R}(512)$ & $\mathrm{R}(32)$ & $R(256)$ & $\mathrm{R}(512)$ & $\mathrm{R}$ & $\mathrm{R}$ & $\mathrm{R}$ & $\mathrm{R}$ \\
\hline 51 & NA & + & + & + & $\mathrm{R}(128)$ & $R(512)$ & $\mathrm{R}(128)$ & $R(512)$ & $R(16)$ & $\mathrm{R}$ & $\mathrm{S}$ & $\mathrm{S}$ & $\mathrm{S}$ \\
\hline 52 & NA & + & + & + & $\mathrm{R}(128)$ & $\mathrm{R}(128)$ & $\mathrm{R}(128)$ & $R(256)$ & $\mathrm{R}(16)$ & $\mathrm{R}$ & $\mathrm{R}$ & $\mathrm{R}$ & $\mathrm{S}$ \\
\hline ATCC 25923 & ATCC & + & + & + & $\mathrm{S}$ & $\mathrm{S}$ & $\mathrm{S}$ & $\mathrm{S}$ & $\mathrm{S}$ & $\mathrm{S}$ & $\mathrm{S}$ & $\mathrm{S}$ & $\mathrm{S}$ \\
\hline
\end{tabular}

+ Represents the presence of gene(s); * Represents strains carrying a plasmid; $\mathrm{R}=$ Resistant; $\mathrm{S}=$ Sensitive

$\mathrm{Cip}^{\mathrm{a}}$, ciprofloxacin; Enr, Nor, norfloxacin; Lev, levofloxacin; enrofloxacin; Oxa, oxacillin supplemented with $2 \% \mathrm{NaCl}$; Tet, tetracycline; Ery, erythromycin; Gen, gentamicin; and Kan, kanamycin. The numbers in parentheses indicate the MIC values. 


\subsection{PCR analysis of FQ resistance and efflux pump genes}

Analysis of the PCR product sizes on $0.8 \%$ agarose gels indicated the expected size amplicons (Fig. 1) for FQ resistance genes, gyrA (493 bp), gyrB (332 bp), grlA (1187 bp), $\operatorname{grlB}(1308 \mathrm{bp})$, the norA promoter region (449 bp) and efflux pump genes, norA (726 bp), norB (117) bp), norC (216 bp), $m d e A$ (677 bp), and mepA (718 bp). Most of the isolates contained the efflux pump genes listed above (Table 2).

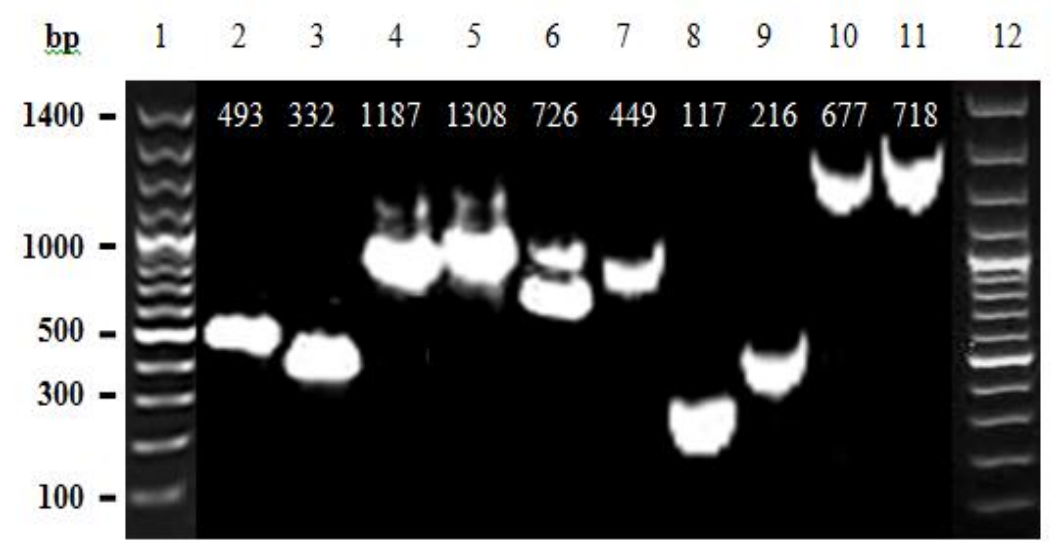

Fig. 1. Panel A; PCR amplification of fluoroquinolone resistance genes from $S$. aureus isolate 10 Lanes 1 and 12, 100 bp-ladder; lane 2, gyrA; lane 3, gyrB; lane 4, grlA; lane 5, grlB; and efflux pump genes; lane 6, norA; lane 7, norA promoter; lane 8, norB; lane 9, norC; lane 10, mdeA; lane 11, mepA.

Table 2. List of efflux pump genes in clinical $S$. aureus isolates

\begin{tabular}{lccccccccccc}
\hline Genes & 10 & 30 & 33 & 35 & 41 & 42 & 49 & 50 & 51 & 52 & ATCC25923 \\
\hline norA & + & + & + & + & + & + & + & + & + & + & - \\
norB & + & + & + & + & + & + & + & + & + & + & - \\
nor $C$ & + & + & + & + & + & + & + & + & + & + & - \\
mdeA & + & + & + & + & + & + & + & + & + & + & - \\
mepA & + & + & + & + & + & + & + & + & + & + & - \\
\hline
\end{tabular}

+ Represents presence of gene, - represents absence of genes

\subsection{Effect of efflux pump inhibitors on ethidium fluorescence and MIC}

A measurement of the efflux pump activity in all the fluoroquinolone-resistant $S$. aureus isolates resulted in an increased $\mathrm{EtBr}$ uptake and enhanced fluorescence intensity in the presence of efflux pump inhibitors, CCCP and RES (representative data shown for isolates 10 and 50) but didn't change for the susceptible $S$. aureus strain ATCC 25923 (Fig. 2). Moreover, the MIC of ciprofloxacin and norfloxacin changed several fold in the presence of CCCP and RES (Table 3). The MIC of ciprofloxacin was reduced 4 to 16 fold for all the isolates in the presence of CCCP and 2 to 8 -fold in the presence of RES. Likewise, the MIC of norfloxacin was reduced 4 to 64-fold in the presence of CCCP and 4 to 16 -fold in the presence of RES. 


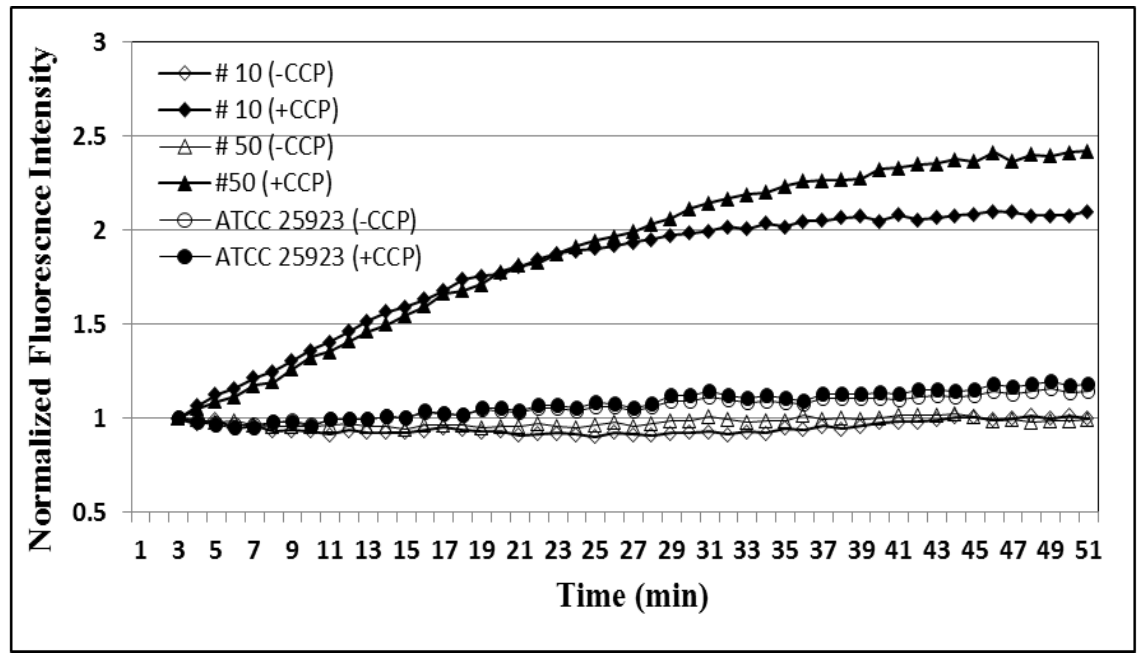

Fig. 2. Demonstration of efflux pump activity in clinical $S$. aureus isolates 10 and 50 and ATCC 25923 by ethidium bromide uptake. The assay was run at $37^{\circ} \mathrm{C}$ in the presence of $\mathrm{EtBr}$, at final concentration of $2.5 \mathrm{mg} / \mathrm{L}$ and in the absence or presence of the efflux pump inhibitor CCCP, at half of its MIC (0.36 $\mathrm{g} / \mathrm{L}$ ). Fluorescence was read every min with excitation at $530 \mathrm{~nm}$ and emission at $600 \mathrm{~nm}$.

Table 3. Effect of efflux pump inhibitors on MICs of fluoroquinolones in clinical S. aureus isolates

\begin{tabular}{cllllll}
\hline Isolate \# & CIP & CIP + CCCP & CIP + RES & NOR & NOR + CCCP & NOR + RES \\
\hline $\mathbf{1 0}$ & $\mathrm{R}(64)$ & $(16)-4 \mathrm{X}$ & $(32)-2 \mathrm{X}$ & $\mathrm{R}(32)$ & $(4)-8 \mathrm{X}$ & $(8)-4 \mathrm{X}$ \\
$\mathbf{3 0}$ & $\mathrm{R}(128)$ & $(32)-4 \mathrm{X}$ & $(64)-2 \mathrm{X}$ & $\mathrm{R}(64)$ & $(16)-4 \mathrm{X}$ & $(32)-4 \mathrm{X}$ \\
$\mathbf{3 2}$ & $\mathrm{R}(512)$ & $(64)-8 \mathrm{X}$ & $(64)-8 \mathrm{X}$ & $\mathrm{R}(128)$ & $(16)-8 \mathrm{X}$ & $(32)-8 \mathrm{X}$ \\
$\mathbf{3 5}$ & $\mathrm{R}(512)$ & $(32)-16 \mathrm{X}$ & $(128)-4 \mathrm{X}$ & $\mathrm{R}(256)$ & $(32)-8 \mathrm{X}$ & $(64)-4 \mathrm{X}$ \\
$\mathbf{4 1}$ & $\mathrm{R}(256)$ & $(64)-4 \mathrm{X}$ & $(128)-2 \mathrm{X}$ & $\mathrm{R}(256)$ & $(8)-32 \mathrm{X}$ & $(32)-8 \mathrm{X}$ \\
$\mathbf{4 2}$ & $\mathrm{R}(256)$ & $(32)-8 \mathrm{X}$ & $(64)-4 \mathrm{X}$ & $\mathrm{R}(512)$ & $(8)-64 \mathrm{X}$ & $(32)-16 \mathrm{X}$ \\
$\mathbf{4 9}$ & $\mathrm{R}(512)$ & $(64)-8 \mathrm{X}$ & $(128)-4 \mathrm{X}$ & $\mathrm{R}(128)$ & $(4)-32 \mathrm{X}$ & $(16)-8 \mathrm{X}$ \\
$\mathbf{5 0}$ & $\mathrm{R}(256)$ & $(32)-4 \mathrm{X}$ & $(128)-2 \mathrm{X}$ & $\mathrm{R}(512)$ & $(16)-32 \mathrm{X}$ & $(64)-8 \mathrm{X}$ \\
$\mathbf{5 1}$ & $\mathrm{R}(128)$ & $(16)-8 \mathrm{X}$ & $(32)-4 \mathrm{X}$ & $\mathrm{R}(512)$ & $(16)-32 \mathrm{X}$ & $(64)-8 \mathrm{X}$ \\
$\mathbf{5 2}$ & $\mathrm{R}(128)$ & $(16)-8 \mathrm{X}$ & $(32)-4 \mathrm{X}$ & $\mathrm{R}(128)$ & $(16)-8 X$ & $(32)-4 \mathrm{X}$ \\
& & & & & & \\
\hline
\end{tabular}

$\mathrm{R}=$ Resistant; Values in parentheses correspond to MIC values of ciprofloxacin and norfloxacin in the absence or presence of CCCP and RES. The numbers followed by X represent the fold decrease in ciprofloxacin norfloxacin MICs in the presence of CCCP and RES at concentrations of 0.36 and $5 \mathrm{mg} / \mathrm{L}$, respectively.

\subsection{RT-qPCR analysis of efflux pump genes}

Transcriptomic expression by RT-qPCR analysis of the efflux pump genes, norA, nor $B$, nor $C$, mdeA and mepA, after induction with $\mathrm{EtBr}$ indicated overexpression of norA, norB, and norC pumps (Fig. 3). There was no observed overexpression of терA (data not shown) in any of the isolates tested, and $m d e A$ was overexpressed significantly in isolates 30, 32, 35, and 41 (data shown for isolate 30). The efflux pump gene norA showed an increase in expression of 20, 17.5, $5.5,16$, and 12-fold in isolates $30,42,50,51$ and 52, respectively (Fig. 3). The expression profiles of these genes in isolate 10 and the FQ-susceptible S. aureus strain ATCC 25923 did not show any change (data shown for isolate 10). The overexpression of the efflux pump gene norB was $>3,8$, and 2-fold in isolates 30,42 , and 51 , respectively. No overexpression of norB was observed in isolates 10,50 and 52 . A 9.5, 7.5, 2.5 and 2fold increase in the expression level of norC gene was observed in isolates $30,42,51$, and 52. No overexpression of norC gene was observed in isolates 10 and 50 .

Volume 2, Issue 2 


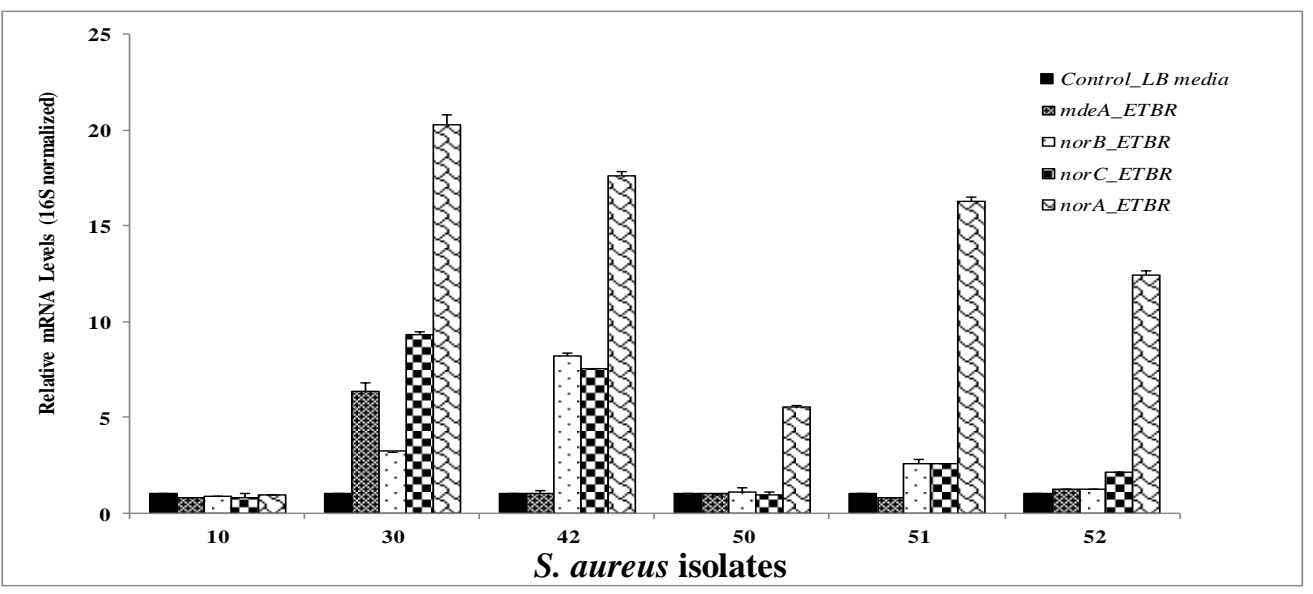

Fig. 3. RT-qPCR amplification of efflux pump genes from representative $S$. aureus isolates after exposure to $\mathrm{EtBr}(2.5 \mathrm{mg} / \mathrm{L})$. The relative expression levels of $\operatorname{norA}$, norB, norC, and $\mathrm{mdeA}$ were calculated using the comparative cycle threshold $\left(\mathrm{C}_{\mathrm{T}}\right)$ method and 16S rRNA expression levels were used for normalization.

\subsection{Genetic mutation analysis}

Sequence analysis of the QRDR of the target gene gyrA indicated a single point mutation (S84L) in isolates 30, 42, and 49, and a double point mutation (S84L and G106D) in isolates 10, 32, 35, and 41. Three of the isolates $(50,51$, and 52) did not have any mutation in gyrA (Table 4). None of the isolates had any mutation in $g y r B$ (data not shown). For grlA and $\operatorname{grlB}$, most of the isolates had a single mutation in $\mathrm{grlA}$ and double mutation in $g r l B$. The only isolate that had a double point mutation in $\operatorname{grlA}$ (S80F, E84G) was isolate 41; the other five isolates, that had a single point mutation in grlA included isolates 42 and 49 (S80Y), 30 (S81P), 32 (E84G), and 50 (P144S). Those that carried double point mutations in the
grlB gene included isolates 10, 51, 52 (E422D, E596D), isolate 35 (S437P, D646Y), and isolate 50 (E422D, V615I). Isolates $10,35,51$, and 52 had no mutation in the grlA gene and isolates 30, 32, 41, 42, and 49 had no mutation in $\mathrm{grlB}$. Sequence analysis of norA revealed that all the isolates lacked the $f l q B$ mutation as well as large deletions in the norA promoter (Fig. 4). Five of the 10 isolates had a deletion of nucleotide (A) upstream of a putative Fur-binding box when their sequences were compared to $S$. aureus ATCC 25923 (Fig. 4). Of these five isolates, isolate 10 had a novel point mutation (V371I) within the norA coding region and the other four isolates $(30,32,35$, and 41) had another novel point mutation (G291D).

Table 4. QRDR mutations in clinical $S$. aureus isolates

\begin{tabular}{|c|c|c|c|c|c|c|}
\hline Isolate & Cip MIC & nor $A^{1}$ & nor $A$ promoter & $g y r A^{2}$ & $g r L A^{3}$ & $\operatorname{gr} l B^{4}$ \\
\hline 10 & $>64$ & Val371Ile & none & Ser84Leu, Gly106Asp & None & Glu422Asp, Glu596Asp \\
\hline 30 & $>128$ & Gly291Asp & $\operatorname{del}(A)$ & Ser84Leu & Ser81Pro & None \\
\hline 32 & $>512$ & Gly291Asp & $\operatorname{del}(A)$ & Ser84Leu, Gly106Asp & Glu84Gly & None \\
\hline 35 & $>256$ & Gly291Asp & $\operatorname{del}(A)$ & Ser84Leu, Gly106Asp & None & Ser437Pro, Asp646Tyr \\
\hline 41 & $>128$ & Gly291Asp & $\operatorname{del}(\mathrm{A})$ & Ser84Leu, Gly106Asp & Ser80Phe, Glu84Gly & None \\
\hline 42 & $>256$ & None & $\operatorname{del}(\mathrm{A})$ & Ser84Leu & Ser80Tyr & None \\
\hline 49 & $>256$ & None & $\operatorname{del}(\mathrm{A})$ & Ser84Leu & Ser80Tyr & None \\
\hline 50 & $>128$ & None & none & None & Pro144Ser & Glu422Asp, Val615Ile \\
\hline 51 & $>128$ & None & none & None & None & Glu422Asp, Glu596Asp \\
\hline 52 & $>128$ & None & none & None & None & Glu422Asp, Glu596Asp \\
\hline
\end{tabular}

GenBank accession numbers: ${ }^{1}$ D90119; ${ }^{2} \mathrm{X} 71437 ;{ }^{3}$ D67074; ${ }^{4} \mathrm{D} 67075$ 


ATCC25923_norAp
10-NorAp
30-NorAp
32-NorAp
35-NorAp
41-NorAp
49-NorAp
50-NorAp
51-NorAp
52-NorAp

ATCC25923 norAp

10-NorAp

30-NorAp

32-NorAp

35-NorAp

41-NorAp

49-NorAp

50-NorAp

51-NorAp

52-NorAp

ATCC25923 norAp

10-NorAp

30-NorAp

32-NorAp

35-NorAp

41-NorAp

49-NorAp

50-NorAp

51-NorAp

52-NorAp

ATCC25923 norAp

10-NorAp

30-NorAp

32-NorAp

35-NorAp

41-NorAp

49-NorAp

50-NorAp

51-NorAp

52-NorAp
Fur-binding box
ATATGTCATT AATTATACAA TTAAATGGAA
AATAGTGATA ATTACAAAGA

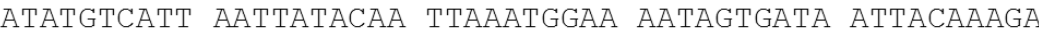

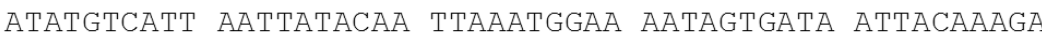 ATAtgtcAtт AATtATACAA tTAAAtgGAA AAtAgtgAtA ATtAcAAAgA

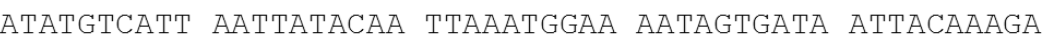 ATATgtcAtT AATtATACAA tTAAATgGAA AAtAgtgAtA ATtAcAAAgA

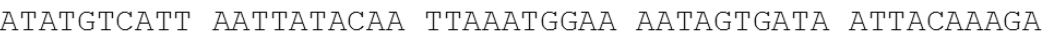

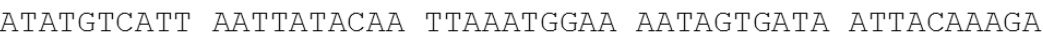

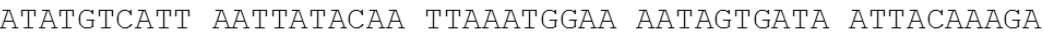

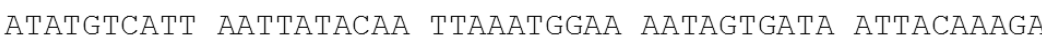

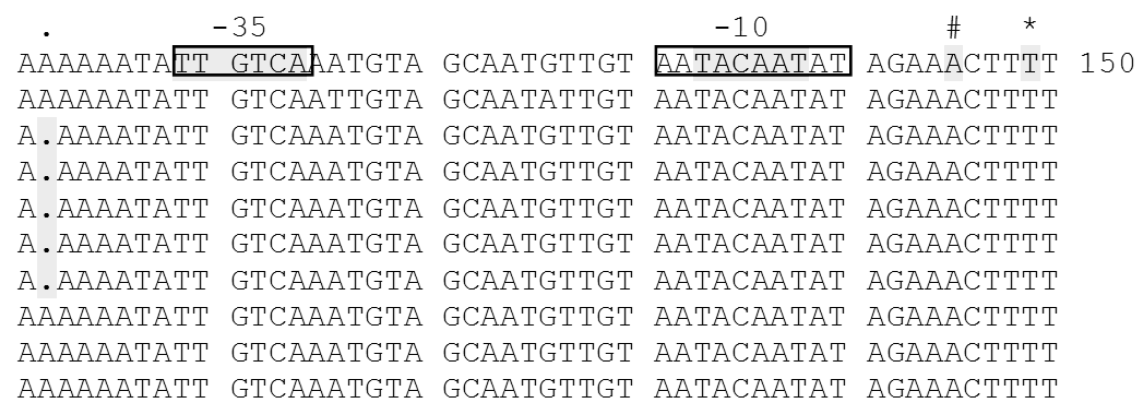

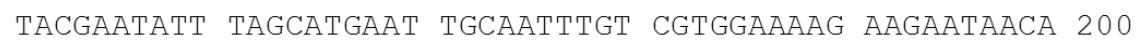

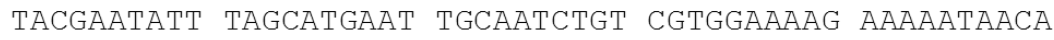
tACGAATAT tAgCATgAat tgcAAtctgt CGTGgAAAAg AAgAAtAACA

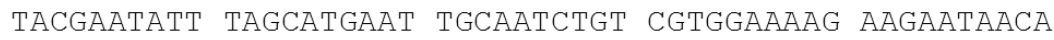
tACGAATAT TAgCATGAAT tgCAATCTgT CGTGgAAAA AAgAATAACA

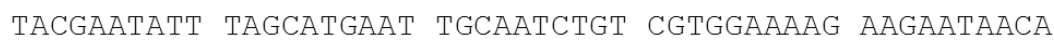

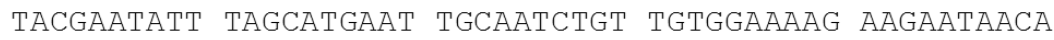
TACGAATAT TAgCATGAAT tgCAATtTgt CGTGgAAAA AAgAAtAACA

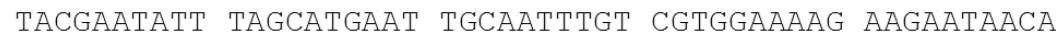

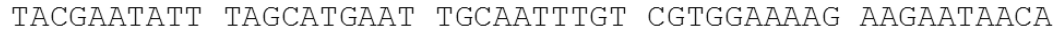

Fig. 4. Alignment of the norA promoter sequences of clinical $S$. aureus isolates with that of ATCC 25923. The consensus sequences -10, -35 and the Shine-Dalgarno (SD) for ATCC 25923 are boxed, including the putative Fur-binding box. * represents the nucleotide $(\mathrm{T})$ of the $f l q B$ mutation; - represents the deletion; \# marks the ATCC 25923 transcription initiation site, and $\rightarrow$ indicates the nor $A$ initiation start codon.

\subsection{In silico structural analysis of novel (NorA) mutations}

A structural analysis of the wild type and mutated NorA proteins from $S$. aureus shows 12 TM helices (TMs 1-12), which form the $\mathrm{N}$ - (residues 3-173) and C-domains (residues 203-375) of the TM core (Fig. A).
There is a central cavity of about $5,007 \AA^{3}$ in the TM core between the $\mathrm{N}$ - and $\mathrm{C}$-domains connected by a 26-residue linker (residues 175-201) with a single $\alpha$-helix and an extended loop. The amino acid residues and

Volume 2, Issue 2 
their TM helices responsible for substrate binding are highlighted in the secondary and tertiary structures with green color. The two novel mutations observed in the NorA (G291D and V371I) are located on the Cdomain. The substitution, G291D, is located in the loop joining the two $\alpha$-helices H9 (position 270 to 288) and H10 (293 to 314), and the V371I mutation is on the $\alpha$-helix H12 (355 to 375 ), surrounded by the two $\alpha-$ helices, H7 (205 to 227) and H9 (270 to 288). In the wild-type NorA protein, the side chain of Gly-291 is totally exposed on the enzyme surface with solvent accessibility of about $42 \%$ and the Val-371 residue shows about $13 \%$ solvent accessibility. In the mutated version of the NorA $\mathrm{A}_{\mathrm{G} 291 \mathrm{D}}$, the overall stability changed as per calculation, using the atom and torsion angle potentials, and increased with the predicted $\Delta \Delta \mathrm{G}$ of 0.48 $\mathrm{kcal} / \mathrm{mol}$. On the other hand, V371I substitution decreased stability by $-1.12 \mathrm{kcal}$ /mol. Despite the introduction of an intermediately sized, negatively charged polar side chain of aspartic acid instead of glycine (proton), the mutant NorA $\mathrm{A}_{\mathrm{G} 291 \mathrm{D}}$ keeps the contacts, including the hydrogen bonds, of the wild-type, whereas in NorA $A_{V 371 I}$ mutant, no change in contacts was observed (Fig. 5B).

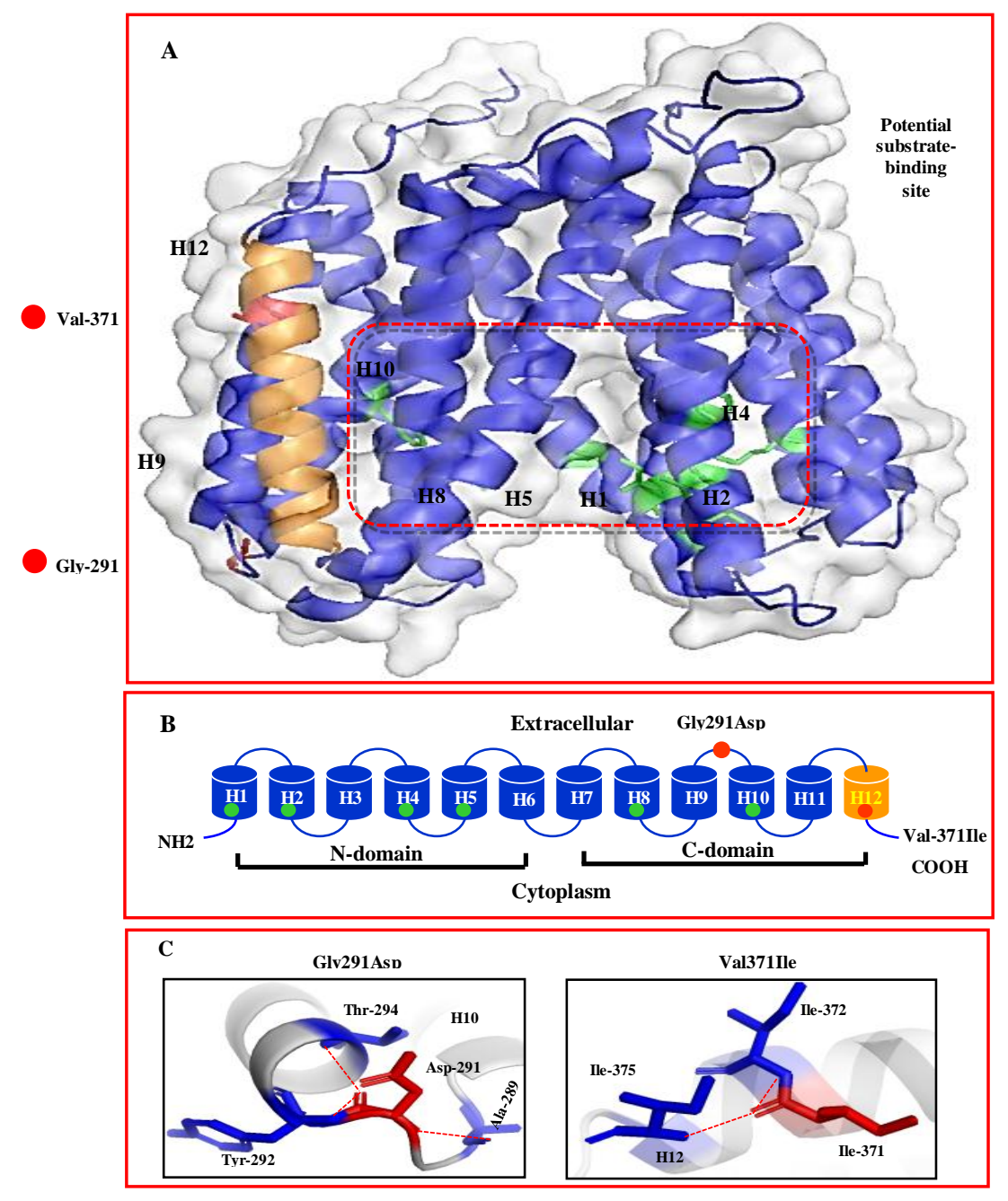

Fig. 5. (A) Three-dimensional homology model and cartoon representation of the secondary structure of the wild-type NorA. The mutation sites are indicated with red dots. The red dashed line indicates a potential active site with the residues (green color) responsible for substrate binding. (B) Helix and loop model of NorA, showing Gly291Asp and Val371Ile locations, together with the residues (green dots) responsible for substrate binding. (C) Structural representation of hydrogen bonds of the Asp-291 and Ile-371 residues with their neighboring amino acids.

Copyright 2015 KEI Journals. All Rights Reserved

Volume 2, Issue 2 


\subsection{Discussion}

FQ resistance in $S$. aureus has mainly been attributed to mutations in the QRDRs of the DNA gyrase and topoisomerase IV genes, gyrA, gyrB, grlA, and $\operatorname{grlB}[4,5,28,31]$. These mutations decrease the affinity of FQ drugs for the target enzyme-DNA complexes, consequently resulting in high level FQ resistance. Our study identified mutations within the QRDR that have been associated with FQ resistance in the past, such as S84L and G106D in gyrA and S80Y or E84G in grlA, and exhibited ciprofloxacin resistance with MICs ranging from 64-512 $\mathrm{mg} / \mathrm{L}$. Double mutations in $\mathrm{grlB}$ (E422D and E596D), previously reported by Sanfilippo et al [28] in methicillin-sensitive $S$. aureus (MSSA) isolates, were also detected. Additional mutations resulting in V615I, S437P, and D646Y substitutions were also observed. In addition to the mutations in gyrase and topoisomerase genes, the contribution of efflux pumps that confer resistance to multiple antimicrobial agents in $S$. aureus has also been reported $[6,18,24$, 27]. We observed the presence of efflux pump activity in all $S$. aureus isolates by an increased $\mathrm{EtBr}$ uptake and a reduction of antibiotic MICs in the presence of efflux pump inhibitors, CCCP and RES, as reported earlier $[8,24,30]$. A two to sixty four-fold reduction in FQ MICs after addition of the EPIs further confirmed the presence of active efflux pump activities in these isolates. CCCP inhibited the efflux pump activity better than RES. Although different EPIs have been reported to block the ATP-binding site of the norA gene resulting in the inhibition of antimicrobial efflux and improved antimicrobial efficacy, their binding scores did not correlate with the level of reduction in MICs [29]. Efflux pump activity alone is not responsible for fluoroquinolone resistance, mutations/ deletions in the QRDR, norA coding and promoter regions also contribute to high level of FQ resistance [8, 12, 18, 24, 26]. Therefore, the addition of CCCP and RES did not completely inhibit FQ resistance. Mutations in the QRDR have been reported to confer resistance up to $32 \mathrm{mg} / \mathrm{L}$, above which the resistance is mainly driven by efflux activity [5], encoded either by the EP genes norA or mepA or by the overexpression of two or more EP genes, mostly norA and norC [8]. The isolates used in this study appear to predominantly overexpress norA and norC genes concurrently, with a few isolates overexpressing norB, nor $C$, and $m d e A$ genes. While nine out of ten FQ-resistant isolates overexpressed the nor $A$ gene, isolate $10 \mathrm{did}$ not. A comparison of the norA sequence data from isolates 10, 51, and 52 revealed that isolate 10 had a novel point mutation V371I in norA that was absent in isolates 51 and 52, indicating a possible role of V371I mutation in the regulation of norA. Additionally, isolate 10 also had double point mutations in the gyrA gene (Ser84Leu, Gly106Asp); it is possible that these mutations play a role in the regulation of norA. However, isolates 50, 51 , and 52 that did not have these mutations but still overexpressed the norA gene clearly suggesting that the V371I rather than Ser84Leu, Gly106Asp mutations played an important role in the regulation of norA gene expression. Some of the isolates, namely 30 , 32,35 , and 41 had the deletion of nucleotide ' $A$ ' upstream of a putative fur-binding box and a novel point mutation G291D in the norA gene that were unique and not reported earlier. The deletion of sequence upstream of a putative Fur-binding box has been shown to have positive regulation of norA expression [12]. Another study [9] demonstrated that the norA transcription was positively regulated by fur (ferric uptake regulator) and its deletion resulted in a compromised norA transcription and reduced resistance to quinolone. The observed overexpression of norA, norB, norC, and $m d e A$ genes in isolates $30,32,35$, and 41 that had nucleotide " $A$ ' deletion and carried G291D mutation, and the overexpression of only norA, nor $B$, nor $C$, but not the mdeA gene in isolates 42 and 49 that had only a deletion of nucleotide " $A$ " and no G291D mutation, suggests that G291 is possibly 
involved in the positive regulation of $m d e A$ gene. Moreover, since norB and norC overexpressed in isolates $30,32,35,41,42$, and 49 that harbored a G291D mutation and nucleotide " $A$ " deletion but not in their absence in isolates $10,50,51$, and 52 , it is possible that the regulation of norB and norC is also controlled by G291D mutation and nucleotide "A" deletion. Furthermore, isolate 10 that had V371I mutation exhibited a relatively low FQ resistance compared to other isolates. In silico modeling revealed a decrease in the stability of norA in isolate 10 by $-1.12 \mathrm{kcal} / \mathrm{mol}$ may explain the relatively low FQ resistance observed in that isolate compared to isolates $30,32,35$, and 41 , that harbored a G291D mutation in the coding region. G291D confers a stabilizing effect due to a predicted $\Delta \Delta \mathrm{G}$ of $0.48 \mathrm{kcal} / \mathrm{mol}$ and its presence along with a deletion in the promoter region of the norA gene, possibly results in a fitness advantage for G291D clones. Of the two novel point mutations observed within the norA gene in this study, G291D is distant from the putative substratebinding sites but V371 is within the second binding site known as "Walker B" site, it is neither involved in hydrogen bonding nor hydrophobic interactions [32]. Therefore, a V371I mutation probably exhibited no change in the expression of norA, norB, nor $C$, and $m d e A$. In conclusion, our findings not only correlate with the earlier studies [6, $8,10,12,26]$ but also describe two novel point mutations in the norA gene and the deletion of nucleotide " $A$ " upstream of a putative fur-binding box and their possible role in the regulation of efflux pump genes norA, norB, nor $C$, and $m d e A$. The role of these mutations in efflux pump-mediated FQ resistance $S$. aureus is quite evident and will be useful in potential development of effective EPIs for better mitigation and treatment strategies of multidrug-resistant pathogens in hospitals.

\section{Acknowledgements}

Drs. Bernard Marasa and Saira Iram contributed equally for the data presented in this manuscript. The authors thank Drs. John
Sutherland and Steven Foley of the Division of Microbiology, NCTR, for critical reading and evaluation of the manuscript. Views presented in this paper do not necessarily reflect those of the FDA.

\section{Funding}

The work was supported by the National Center for Toxicological Research, US Food and Drug Administration.

\section{Competing Interests}

None declared

\section{Ethical approval}

Not require

\section{References}

[1] Bauer AW, Kirby WM, Sherris JC, Turck M. Antibiotic susceptibility testing by a standardized single disk method. Am. J. Clin. Pathol. 1966;45: 493-96.

[2] Berger-Bachi B, Rohrer S. Factors influencing methicillin resistance in staphylococci. Arch. Microbiol. 2002; 178:165-71.

[3] Clinical and Laboratory Standards Institute (CLSI). Methods for dilution antimicrobial susceptibility tests for bacteria that grow aerobically; approved standard: ninth edition, M07-A9. Wayne, PA: CLSI; 2012.

[4] Costa SS, Falcao C, Viveiros M, Machado D, Martins M, Melo-Cristino J, Amaral L, Couto I. Exploring the contribution of efflux on the resistance to fluoroquinolones in clinical isolates of Staphylococcus aureus. BMC Microbiol. 2011;11: 241.

[5] Costa SS, Viveiros M, Amaral L, Couto I. Multidrug Efflux Pumps in 
Staphylococcus aureus: an Update. Open Microbiol. J. 2013;7:59-71.

[6] Couto I, Costa SS, Viveiros M, Martins M, Amaral L. Efflux-mediated response of Staphylococcus aureus exposed to ethidium bromide. J. Antimicrob. Chemother. 2008;62:504-13.

[7] De Lencastre H, Oliveira D, Tomasz A. Antibiotic resistant Staphylococcus aureus: a paradigm of adaptive power. Curr. Opin. Microbiol. 2007;10:428-35.

[8] De Marco CE, Cushing LA, FrempongManso E, Seo SM, Jaravaza TA, Kaatz GW. Efflux-related resistance to norfloxacin, dyes, and biocides in bloodstream isolates of Staphylococcus aureus. Antimicrob. Agents Chemother. 2007;51:3235-39.

[9] Deng X, Sun F, Ji Q, Liang H, Missiakas D, Lan L, He C. Expression of multidrug resistance efflux pump gene norA is iron responsive in Staphylococcus aureus. J. Bacteriol. 2012;194:1753-62.

[10] Fournier B, Truong-Bolduc QC, Zhang $\mathrm{X}$, Hooper DC. A mutation in the 5' untranslated region increases stability of norA mRNA, encoding a multidrug resistance transporter of Staphylococcus aureus. J. Bacteriol. 2001;183:2367-71.

[11] Gordon RJ, Lowy FD. Pathogenesis of methicillin-resistant Staphylococcus aureus infection. Clin. Infect. Dis. 2008; 46 Suppl 5:S350-59.

[12] Noguchi N, Okada H, Narui K, Sasatsu M. Comparison of the nucleotide sequence and expression of norA genes and microbial susceptibility in 21 strains of Staphylococcus aureus. Microb. Drug Res. 2004;10:197-203.

[13] Hirokawa T, Boon-Chieng S, Mitaku S. SOSUI: classification and second-ary structure prediction system for membrane proteins. Bioinformatics. 1998;14:378-379.

[14]Jiang D, Zhao Y, Wang X, Fan J, Heng J, Liu X, Feng W, Kang X, Huang B, Liu J, Zhang XC. Structure of the YajR transporter suggests a transport mechanism based on the conserved motif A. Proc. Natl. Acad. Sci. USA. 2013;110:14664-69.

[15] Karczmarczyk M, Martins M, Quinn T, Leonard N, Fanning S. Mechanisms of fluoroquinolone resistance in Escherichia coli isolates from foodproducing animals. Appl. Environ. Microbiol. 2011;77:7113-20.

[16]Kelley LA, Sternberg MJ. Protein structure prediction on the Web: a case study using the Phyre server. Nat. Protoc. 2009;4:363-71.

[17] Krogh A, Larsson B, von Heijne G, Sonnhammer EL. Predicting transmembrane protein topology with a hidden Markov model: application to complete genomes. J. Mol. Biol. 2001; 305:567-80.

[18] Kwak YG, Truong-Bolduc QC, Bin Kim $\mathrm{H}$, Song KH, Kim ES, Hooper DC. Association of norB overexpression and fluoroquinolone resistance in clinical isolates of Staphylococcus aureus from Korea. J. Antimicrob. Chemother. 2013; 68:2766-72.

[19]Langmann T, Mauerer R, Zahn A, Moehle C, Probst M, Stremmel W, Schmitz G. Real-time reverse transcription-PCRexpression profiling of the complete human ATP-binding cassette transporter superfamily in various tissues. Clin. Chem. 2003;49: 230-38.

[20]Louie A, Brown DL, Liu W, Kulawy $\mathrm{RW}$, Deziel MR, Drusano GL. In vitro infection model characterizing the effect of efflux pump inhibition on prevention 
of resistance to levofloxacin and ciprofloxacin in Streptococcus pneumoniae. Antimicrob. Agents Chemother. 2007;51:3988-00.

[21]Lowy FD. Staphylococcus aureus infections. N. Engl. J. Med. 1998; 339:520-32.

[22] Lowy FD. Antimicrobial resistance: the example of Staphylococcus aureus. J. Clin. Invest. 2003;111:1265-73.

[23] Marquez B. Bacterial efflux systems and efflux pumps inhibitors. Biochimie. 2005;87:1137-47.

[24] Martins M, Viveiros M, Couto I, Costa SS, Pacheco T, Fanning S, Pages JM, Amaral L. Identification of efflux pumpmediated multidrug-resistant bacteria by the ethidium bromide-agar cartwheel method. In Vivo. 2011;25:171-78.

[25] Ng EY, Trucksis M, Hooper DC. Quinolone resistance mediated by norA: physiologic characterization and relationship to $f l q B$, a quinolone resistance locus on the Staphylococcus aureus chromosome. Antimicrob. Agents Chemother. 1994;38:1345-55.

[26] Ohshita Y, Hiramatsu K, Yokota T. A point mutation in norA gene is responsible for quinolone resistance in Staphylococcus aureus. Biochem. Biophys. Res. Commun. 1990;172: 1028-34.

[27] Patel D, Kosmidis C, Seo SM, Kaatz GW. Ethidium bromide MIC screening for enhanced efflux pump gene expression or efflux activity in Staphylococcus aureus. Antimicrob. Agents Chemother. 2010;54:5070-73.
[28] Sanfilippo CM, Hesje CK, Haas W, Morris TW. Topoisomerase mutations that are associated with high-level resistance to earlier fluoroquinolones in Staphylococcus aureus have less effect on the antibacterial activity of besifloxacin. Chemotherapy. 2011; 57: 363-71.

[29] Sanger F, Coulson AR. A rapid method for determining sequences in DNA by primed synthesis with DNA polymerase. J. Mol. Biol. 1975;94:441-48.

[30] Strahilevitz J, Hooper DC. Dual targeting of topoisomerase IV and gyrase to reduce mutant selection: direct testing of the paradigm by using WCK1734, a new fluoroquinolone, and ciprofloxacin. Antimicrob. Agents Chemother. 2005; 49:1949-56.

[31] Takahashi H, Kikuchi T, Shoji S, Fujimura S, Lutfor AB, Tokue Y, Nukiwa T, Watanabe A. Characterization of gyrA, gyrB, grlA and grlB mutations in fluoroquinolone resistant clinical isolates of Staphylococcus aureus. J. Antimicrob. Chemother. 1998;41:49-57.

[32] Tanaka M, Wang T, Onodera Y, Uchida Y, Sato K. Mechanism of quinolone resistance in Staphylococcus aureus. J. Infect. Chemother. 2000;6:131-39.

[33] Thai KM, Ngo TD, Phan TV, Tran TD, Nguyen NV, Nguyen TH, Le MT. Virtual screening for novel Staphylococcus aureus NorA efflux pump inhibitors from natural products. Med. Chem. 2015;11:135-55. 